\title{
Operational Impact of mHealth Adoption in Clinical Practice
}

\author{
Balaraman Rajan \\ California State University East Bay \\ balaraman.rajan@csueastbay.edu
}

\author{
Saligrama Agnihothri \\ Binghamton University \\ agni@binghamton.edu
}

\begin{abstract}
Chronic conditions place a high cost burden on the healthcare system and deplete the quality of life for millions of Americans. There is significant medical literature that shows that continuous monitoring of patient health at home with the addition of provider support, improves patient health. Digital innovations such as mHealth technology can be used to provide efficient, effective, and patient centered healthcare. However, implementing mHealth technology can significantly change the composition of clinical staff and patient flow. In this paper, we evaluate the tradeoffs of implementing mHealth technology in a clinical practice.
\end{abstract}

\section{Introduction}

In the United States today, about half of all adults have one or more chronic health conditions. Seven of the top ten causes of death in 2014 were chronic diseases [1]. Chronic disease and the delivery of care to manage and treat these conditions may be the most important issue facing our society today $[2,3,4,5]$.

Mobile health (mHealth) is a broad term typically used to describe the use of mobile technologies for the delivery of health care. mHealth could help manage chronic conditions such as hypertension and diabetes. Unlike acute care, chronic healthcare is specially characterized by recursive physician-patient interactions. Mobile technologies can help improve the efficiencies in physician-patient interactions. Instead of infrequent patient visits to the provider's office, patients can measure biometric information (such as blood pressure and blood sugar levels) at home and upload through a smartphone application (see, for example, [6]) to a provider's web-based clinical decision support system. Providers can remotely monitor patient health and can intervene when necessary. This enables greater patient engagement in the care delivery process and prompt diagnosis and treatment of chronic conditions. As a result, the number of unnecessary visits to physicians' offices and emergency departments can be substantially decreased, reducing health care costs.
Telemedicine has the potential to increase provider efficiency [7] and mHealth may become an integral part of the care delivery process. Mobile technologies create the ability to improve patient health and to minimize or even eliminate the need for office visits for the routine management of some of the most common chronic issues [8]. Agnihothri et al. quantify using a Markov model the benefits of mHealth interventions [9].

Unlike acute conditions, many of the most common chronic conditions can be directly attributed to specific patient behaviors. Patient behavior can be composed of first, adherence to the treatment plan, and second, implementation of lifestyle changes that are either preventative or aid in management of the chronic condition such as diet and exercise. Thus, patient education is an important part of patient care. Such tasks could be assigned to physician's support staff such as nurse practitioners, dieticians, and exercise physiologists. By eliminating physicians' unneeded involvement clinicians might be able to spend more time with the patients who need them most.

Thus, adopting mHealth in a clinical practice can significantly change the composition of physician staff and patient flow. This in turn can change the economics associated with care delivery process $[8,10]$. The objective of this paper is to evaluate the impact of implementing mHealth technology on a clinical practice. Specifically, we investigate "when is it economical to switch to an mHealth-based practice from a face-to-face, office visit-based clinical practice?”

\section{Modeling Details}

\subsection{Regular Clinic or Office mode of care}

Before the introduction of mHealth assume a single doctor operating a clinic. She receives payment based on fee for service. Let the reimbursement be given by $p$ for every office visit. In addition to this reimbursement, as a token of serving her patient's needs, we assume the doctor receives a share of the 
patient utility for every patient he serves. This might be a notional feel-good effect of making a patient healthier and better and is therefore independent of number of visits. We assume a linear gain in utility and let the utility per patient be given by $p_{u}$. (List of notations is provided in Table 1)

We assume the clinic serves different kinds of patients and the total patient panel size is N. For simplicity we will classify patients into high-risk and low-risk patients with arrival rates $\lambda_{H}$ and $\lambda_{L}$ respectively. These arrivals are over and above the regular periodic visits that the patients are expected to see the doctor for. Let h denote the proportion of highrisk patients in a panel size of $\mathrm{N}$. The high-risk patients have a higher arrival rate (frequent measure of vitals, etc.), that is $\lambda_{H}>\lambda_{L}$. In our initial analysis, we do not assume a priority system in place for clinic operations. Hence the clinic sees a combined arrival rate and the case-mix does not actually play a role.

Table 1: List of Notations:

\section{Reimbursement Model}

$N=$ Panel size

$p=$ the reimbursement for every office visit

$\mu=$ the physician service rate

$p_{u}=$ the utility per patient (the doctor receives a share of the utility for every patient he serves)

$\mathrm{c}=$ reputation cost of making a patient wait in the system for one unit of time

$\lambda_{o}, \lambda_{m}=$ Total average arrival rate for a panel size $\mathrm{N}$ for regular mode and mHealth mode

$\mathrm{n}=$ Expected arrival rate per patient

$\mathrm{h}=$ the proportion of high-risk patients in a panel size of $\mathrm{N}$

$\lambda_{H}=$ arrival rate of high-risk patients

$\lambda_{L}=$ arrival rate of low-risk patients $\left(\lambda_{H}>\lambda_{L}\right)$

$\mathrm{T}=$ Maximum Average delay to get an appointment

\section{Capitation Model}

$\mathrm{p}_{\mathrm{c}}=$ capitation payment per patient per period

$\mathrm{m}=$ number of clinical staff

$c_{p}=$ payroll cost per period per staff

$\mathrm{k}_{1} \mu=$ new physician service rate

$\mu_{s}=$ service rate of clinic staff

$\mathrm{k}_{2} \lambda_{m}=$ the total arrival rate (referral rate) to the doctor

Let the total average arrival rate for a panel size of $\mathrm{N}$ and a case-mix ratio $\mathrm{h}$ be given by $\lambda_{o}=\lambda_{H}+\lambda_{L}$. Since the arrival rate will be a function of the panel size, we have $\lambda_{o}(N)=\lambda(h N)+\lambda((1-h) N)$. Patients incur a disutility (cost) of traveling to the clinic (monetary or otherwise) for every visit and the corresponding share for the doctor can be combined with any marginal cost for the clinic per visit. We can then normalize the combined cost to zero. We assume that the clinic is expected to provide an appointment to patients within $\mathrm{T}$ days on average. Based on the expected standard time for a clinic visit and a dedicated time for non-periodic visits, let the service rate be given by $\mu$. (Note: This implicitly means that the service rate $\mu$ is determined exogenously. The randomness comes from variation in day to day clinic operations.)

The doctor decides on an optimal panel size such that the net revenue for the specialist is maximized. The net revenue per period for the doctor is then given by

$$
\begin{gathered}
\pi\left(\mathrm{N}, \lambda_{o}\right)=p \lambda_{o}+p_{u} N-\frac{c}{\mu-\lambda_{o}} \lambda_{o} \\
\text { s.t. } \frac{1}{\mu-\lambda_{o}} \leq T \text { and } \\
\mu>\lambda_{o}
\end{gathered}
$$

Note that we do not consider the revenue from regular visits in the net revenue function. In expectation terms, this is a fixed amount per period and hence will not change the result of the optimization problem. The term $\frac{c}{\mu-\lambda_{o}}$ can be seen as the cost of reputation if the clinic is very congested with severe appointment delays. We note that the optimal solution will be the same even if we consider only the waiting time (in contrast to the total time) since the service rate is fixed.

If we assume $\lambda_{o}$ to simply be a factor of $\mathrm{N}$ (rather than a function of $\mathrm{N}$ ), that is, each patient is expected to see the doctor $n$ times a year (over and above the periodic "maintenance" visits), we can simplify the net revenue function further and identify the optimal panel size. If we assume $\lambda_{o}=n N$, then the solution to the optimization problem is as follows.

Proposition 1: Let $N^{*}$ denote the optimal panel size. If $n p+p_{u}<\frac{c(1+\mu)^{2}}{\mu^{3}}$, then $N^{*}=0$. If $n p+p_{u} \geq$ $n c T^{2} \mu$, then $N^{*}=\frac{\mu}{n}-\frac{1}{T n}$. Otherwise, $N^{*}=\frac{\mu}{n}-$ $\sqrt{\frac{c \mu}{n\left(n p+p_{u}\right)}}$.

The proof follows from concavity of the objective function and first order conditions. The details are given in the appendix. The first condition ensures that the participation constraint for the provider is satisfied. The second part of the proposition pertains to the case when the service level constraint is binding.

\subsection{Clinic operations with mHealth}

In this section we explore if and when the doctor would adopt mHealth. We use the optimal panel size 
that is in effect before the introduction of mHealth. Given this panel size we optimize the clinic operations (that is, staff level). We then compare the two net revenues in both the modes of care and derive conditions under which mHealth mode is beneficial for the clinic. We identify the range of parameter values where it is optimal to introduce mHealth. Later on we also investigate how the optimal panel size will change with the introduction of mHealth.

Since there are currently limitations with respect to reimbursement for mHealth visits, we explore a capitation-based model in which the doctor gets reimbursed every period for each patient in her panel size. To start with we will assume there are bonuses and/or penalties based on patient health and the payment is conditional on some minimum expectations. We will assume that the doctor satisfies these minimum expectations to obtain the capitation payment $p_{c}$. In the base model we do not identify the optimal penalties and bonuses. We assume them to be exogenous and fixed. This will help us focus on the optimal operation of the clinic for a panel size identified before mHealth adoption.

In order to manage the operations, the doctor employs a few clinical staff. These clinical staff play an active disease management role and they are not simply administrators managing appointments. Their roles could be providing ancillary services (e.g., dieticians), monitoring vitals, etc. Let the payroll cost per period per staff be given by of $c_{p}$ and the number of clinical staff be denoted by $\mathrm{m}$.

We also assume sequential mode of care where all patients are first monitored by clinical staff. On a need basis the clinical staff ask the patients to schedule an appointment with the doctor or put a referral. (Note: Alternatively, if there is an efficient routing system in place that automatically routes arrivals to the appropriate server, the care delivery could be done in parallel instead of sequential.) The mHealth mode of care delivery may potentially increase the efficiency of the doctor since most of the medical service will be provided by her staff and her role will be more of a supervisory role. On the other hand, the doctor may now only deal with sicker and complicated patients after getting triaged by her staff. Dealing with these patients may be difficult and consume more time. We let the new service rate for clinic visits be $k_{1} \mu$, where $k_{1}<>1$. Let the service rate of clinic staff be given by $\mu_{s}$

Even though the arrival rate to the clinic will vary according to the patient-type, case-mix ratio $h$, and patient panel size $\mathrm{N}$, the arrival rate to the doctor will be lower, as much of the treatment can be managed by the clinic staff. Let the total arrival rate (referral rate) to the doctor be given by $\mathrm{k}_{2} \lambda_{m}$, where $k_{2}<1$.
Given the capitation model and a fixed panel size, since payments are fixed, it is enough if we focus on the cost per period of clinic operations for optimization. However, for the sake of consistency, we work with the net revenue as given by

$$
\begin{aligned}
\pi_{m}\left(\mathrm{~N}, \lambda_{m}, m\right)= & p_{c} N-\frac{c}{k_{1} \mu-k_{2} \lambda_{m}} k_{2} \lambda_{m}-c_{p} m \\
& -c_{s} \lambda_{m} W\left(\lambda_{m}, \mu_{s}, m\right)
\end{aligned}
$$

where $W\left(\lambda_{m}, \mu_{s}, m\right)$ has a similar connotation to $\frac{c}{\mu-\lambda_{o}}$ and represents the congestion cost related to the clinic staff. We use the following approximation for the waiting time in queue

$$
\begin{aligned}
& \qquad W\left(\lambda_{m}, \mu_{s}, m\right)=\frac{\rho^{\sqrt{2(m+1)}}}{\lambda_{m}(1-\rho)} \\
& \text { where } \rho=\frac{\lambda_{m}}{m \mu_{s}}[11] .
\end{aligned}
$$

In this case the doctor needs to pick the optimal staff size m. The clinic will then solve the following optimization problem, assuming $\lambda_{m}=n_{m} N$.

$$
\begin{gathered}
\operatorname{Max}_{\mathrm{m}} \pi_{m}\left(\mathrm{~N}, \lambda_{m}(N), m\right) \\
\text { s.t. } W\left(\lambda_{m}(\mathrm{~N}), \mu_{s}, m\right) \leq T_{m} \\
m \mu_{s}>\lambda_{m}(\mathrm{~N})
\end{gathered}
$$

Again, we assume that the staff are expected to provide a response (intervention) to patients within $T_{m}$ days on average. The stability constraints and the service level constraints for the provider are not included in the optimization problem since they are not dependent on the staff size m. However, they are very much relevant and are assumed to be satisfied by the parameter values in the optimization problem. Specifically, the optimization problem is only valid if $\frac{1}{k_{1} \mu-k_{2} \lambda_{m}(\mathrm{~N})} \leq T_{m}$ and $k_{1} \mu>k_{2} \lambda(\mathrm{N})$.

\section{Numerical analysis}

\subsection{Choice of parameters in the base case}

Consider the period to be a day. Two visits is the average number of additional visits to the clinic per patient per year. Assuming the doctor spends about 20 minutes with each patient and is available for 7 hours a day and the doctor allocates $20 \%$ of his time to urgent or non-periodic visits, service rate is then $\mu=$ 4.2 patients per day. We will assume twice the regular rate for the clinical staff on average. $\mu_{s}=42$ patients per day. Assume payroll cost, $c_{p}$, to be 200 dollars per day per staff (based on an annual salary of 73,000 dollars). We assume $p=\$ 50$ per visit, $n=2 / 365$, that is, two non-regular visits per patient per year, 
$p_{u}=1$ per patient per day, $p_{c}=\$ 2$ per patient per day, $k_{1}=1, k_{2}=0.02, c=c_{s}=5$ per day, $T=5$ days, and $T_{m}=1$ day, $n_{m}=1 / 7$, that is, mobile apps are used at least once a week by patients. Since $k_{2}=$ 0.02 , only $2 \%$ of these mobile uploads are referred to the doctor.

\subsection{Pre-mHealth case}

For the base case the optimal panel size is found to be 711 and the total net revenue is 842 per day. We next explore how sensitive the optimal panel size is with respect to the service level requirement $\mathrm{T}$ in Figure 1. We observe that even though the service level constraint is relaxed (higher $\mathrm{T}$ ), the optimal panel size only increases marginally (for $T>4$ ). This is due to the importance the provider gives for her reputation determined by the congestion in her clinic. The behavior of the net revenue is similar.

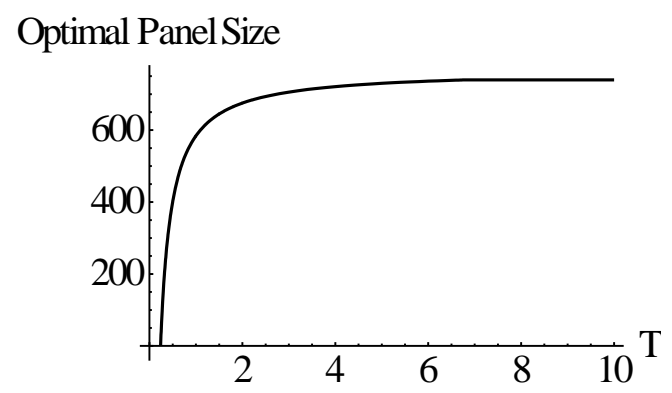

Figure 1: Optimal Panel Size vs. Maximum Average Delay for Appointment

\section{3 mHealth case}

For the base case scenario, the optimal staff size for mHealth mode is 2.7 and the net revenue is 844 per day. The optimal $\mathrm{m}$ is also linearly increasing with $\mathrm{n}_{m}$. We next analyze scenarios or parameter values where the mHealth mode generates more net revenue than the regular office mode.

Figure 2 shows the difference in net revenue for different values of the average number of uploads per patient. We can observe a cut-off value beyond which mHealth mode fares worse than the regular mode. For the base case in Figure 2 we observe that the cut-off value is just above once a week. While the benefit of mHealth comes from the clinical support staff managing most of the care, unexpectedly, mHealth could also make patients seek care more often as the access to health providers becomes easier. If so, then mHealth could potentially increase the load on the clinic, requiring additional staff capacity and hence the net revenue might be lower than that of regular mode.
Clinics should be wary of such patient behavior during the transition phase.

Figure 3 shows the difference in net revenue for different values of the average service rate of clinical staff. The difference in revenue increases with the efficiency of care delivery. Due to the continuous nature of treatment plan, care delivery through mHealth can become efficient and can become patientcentric. With patient-specific data to guide treatment plans, efficiency in care delivery could lead to higher revenue in the case of mHealth. In the base case the service rate of clinic staff is at twice the regular rate of the doctor. While this rate is enough to lead to a higher profit, we also note that further efficiency increase has decreasing returns on the profit.

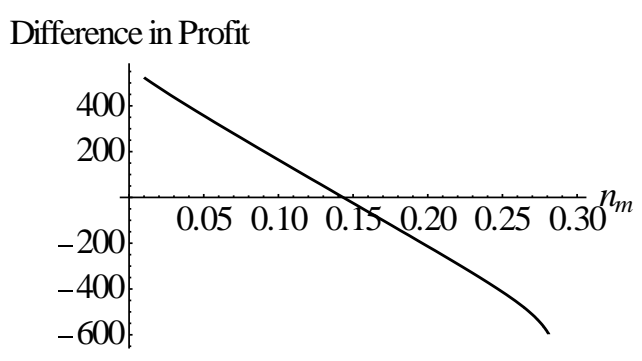

Figure 2: Difference in Total Net Revenue vs. Average Number of Uploads per patient

Difference in Profit

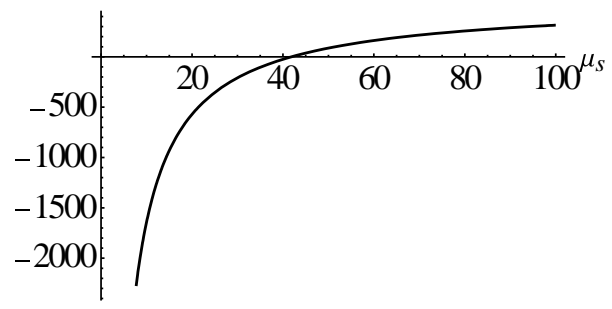

Figure 3: Difference in Total Net Revenue vs. Average Service Rate of Clinic Staff

Figure 4 shows the difference in net revenue for different values of the capitation fee per patient. We can observe a cut-off value only beyond which mHealth mode fares better than the regular mode. This reinforces the key reason why doctors hesitate to venture into mHealth. Even under capitation-based models, if the reimbursement provided to the doctors are insufficient, the incentive to adopt mHealth reduces and the providers would prefer the regular mode of care and a fee-for-service regime. Policy makers need to structure the payments carefully to provide proper incentives for mHealth. 


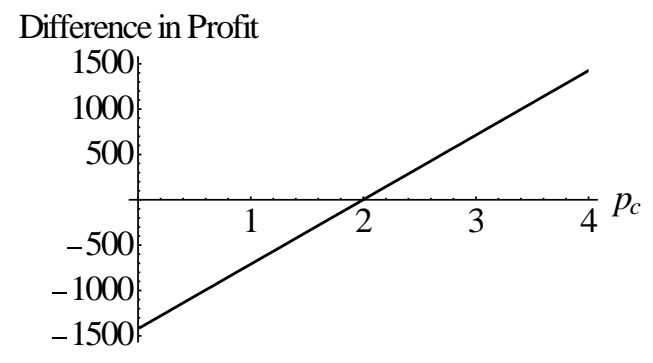

Figure 4: Difference in Total Net Revenue vs. Capitation payment per patient

Figure 5 shows the difference in net revenue for different values of the average service rate of the doctor. We can observe a cut-off value beyond which mHealth mode becomes feasible and fares better than the regular mode. As the service rate increases, the optimal panel size also increases and hence the impact of mHealth increases as well. Similar to our discussion under clinic staff, the availability of regular patientspecific data in the mHealth mode can make care delivery pertinent and efficient. Patients taking control of their health also play a role in increasing the efficiency of the provider.

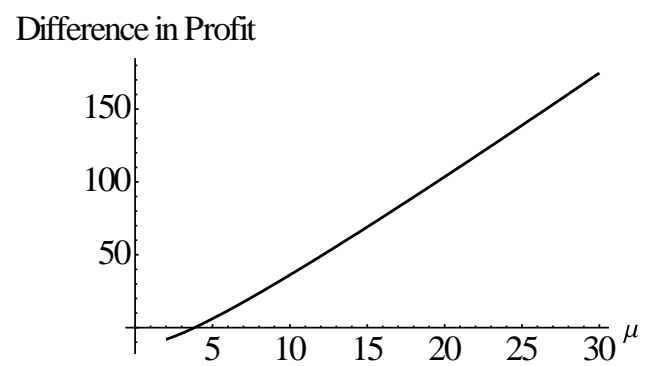

Figure 5: Difference in Total Net Revenue vs. Average Service Rate of Provider

We also analyze how the clinic can choose the optimal panel size in the case of mHealth mode. Finally, we also investigate the differential impact of case-mix on the mHealth mode operations.

\section{Discussion and Conclusion}

Continuous care management has been strongly recommended to decrease the costs associated with chronic care. Healthcare technology including mobile technology can greatly aid in this regard. They can also help in patient-specific care management. In this research we show the conditions under which clinics can adopt mHealth.

While mHealth has shown a lot of promise in patient data collection and giving patient more control, one must also consider the role of providers in the adoption of mHealth. It adds significant burden to the specialists and without proper reimbursement initiatives the benefits of mHealth may not be fully realized. Our analysis brings out the benefits for providers using mHealth. In addition to identifying the key parameters that will influence the adoption of mHealth, our analysis helps in exploring the trade-offs for providers (clinics or physicians) in offering mHealth to its patients. We explore if the government and private insurance companies should play a role for mHealth to be successful and whether such moves will increase social welfare.

\section{References}

[1] Center for Disease Control, 2017. Accessed on October 30, 2017 from

https://www.cdc.gov/chronicdisease/overview/index.htm. [2] Institute of Medicine, 2008. Evidence-based medicine and the changing nature of health care, Washington, D.C.: The National Academies Press.

[3] Milani, R. V., Lavie, C. J., 2015. Health Care 2020: Reeingineering Health Care Delivery to Combat Chronic Disease. The American Journal of Medicine, Volume 128, pp. 337-343.

[4] Wagner, E. H., Austin, B. T., Davis, C., Hindmarsh, M., Schaefer, J., Bonomi, A., 2001. Improving Chronic Illness Care: Translating Evidence into Action. Health Affairs, 20(6), pp. 64-78.

[5] Agnihothri, S. Agnihothri, R. (2018) Application of evidence-based management to chronic disease healthcare: a framework, Management Decision

[6] Banerjee A., Ramanujan R. A., and Agnihothri S., 2016. Mobile Health Monitoring: Analysis of an app in a Diabetes and Hypertension Clinic, Proceedings of the 49th Annual Hawaii International Conference on System Sciences (HICSS), Kauai, HI.

[7] Rajan, B., Tezcan, T., and Seidman, A. (2018). Service Systems with Heterogeneous Customers: Investigating the effect of Telemedicine on Chronic Care, Management Science, Article in Advance, Available Online.

[8] Steinhuble, S.R., Muse, E.D., and Topol, E.J. (2013). "Can Mobile health Technologies Transform Healthcare?" JAMA, Vol. 310 No. 22, pp. 2395-2396.

[9] Agnihothri, S., Cui, L., Delasay, M., and Rajan, B. The Value of mHealth for Managing Chronic Conditions, Health Care Management Science (forthcoming). [10] Liu, N. and D’Aunno, T. (2012). “The productivity and Cost-Efficiency of Models for involving Nurse Practitioners in Primary Care: A Perspective from Queueing Analysis,” Health Services Research, Vol. 47 No. 2, pp. 594-613.

[11] Sakasegawa $H$. An approximation formula $L q \simeq \alpha$. $\rho^{\beta} /(1-\rho)$. Annals of the Institute of Statistical Mathematics. 1977 Dec 1;29(1):67-75. 


\section{Appendix}

Proof of Proposition 1: The objective function is concave in $\mathrm{N}$ since

$$
\frac{d^{2} \pi}{d N^{2}}=-\frac{2 c n^{3} N}{(\mu-n N)^{3}}-\frac{2 c n^{2}}{(\mu-n N)^{2}}<0
$$

Therefore, we can use the first-order conditions to identify the optimal $\mathrm{N}$. The first-order condition yields two roots: $\left\{\frac{\mu}{n}-\frac{\sqrt{c n^{3}\left(n p+p_{u}\right) \mu}}{n^{2}\left(n p+p_{u}\right)}, \frac{\mu}{n}+\frac{\sqrt{c n^{3}\left(n p+p_{u}\right) \mu}}{n^{2}\left(n p+p_{u}\right)}\right\}$. Since $\frac{\mu}{n}+\frac{\sqrt{c n^{3}\left(n p+p_{u}\right) \mu}}{n^{2}\left(n p+p_{u}\right)}$ is infeasible (the second constraint which refers to system stability is not satisfied), only the other root is possible. Only the boundary conditions need to be checked namely, the service constraint and the non-negative objective function. The proposition then follows. 